\title{
Correlative Luminescence and Absorption Spectroscopy from Monolayer WSe2 at the Nanoscale
}

Steffi Woo ${ }^{1}$, Fuhui Shao ${ }^{2}$, Robert Schneider ${ }^{3}$, Ashish Arora ${ }^{3}$, Johann Preuß ${ }^{3}$, Benjamin Carey ${ }^{3}$, Steffen Michaelis $^{3}$, Rudolf Bratschitsch ${ }^{3}$ and Luiz H. G. Tizei ${ }^{4}$

${ }^{1}$ Laboratoire de Physique des Solides/CNRS, Université Paris-Saclay, United States, ${ }^{2}$ Université ParisSaclay, CNRS, Laboratoire de Physique des Solides, United States, ${ }^{3}$ Institute of Physics and Center for Nanotechnology, University of Münster, Germany, ${ }^{4}$ Université Paris-Saclay, CNRS, Laboratoire de Physique des Solides, 91405, Orsay, France, France

Single- and few-layer semiconducting transition metal dichalcogenides (TMDCs) demonstrate to be a fascinating class of two-dimensional (2D) materials that can act as building blocks for van der Waals heterostructures. Standalone, bulk and multi-layered tungsten diselenide (WSe2) are indirect gap semiconductors, while its monolayers exhibit substantial photoluminescence (PL) characteristic of a direct optical bandgap in the visible. At cryogenic temperature, the PL response of monolayer $\mathrm{WSe}_{2}$ is typically composed of the neutral A exciton $\left(\mathrm{X}^{0}\right)$, charged exciton (trion, $\mathrm{X}^{-}$or $\mathrm{X}^{+}$) emission, and a dominant lowerenergy broad emission band [1]. Recently, bright and stable single-photon emission from localized excitons in WSe2 monolayers overlapping a broad emission band has been demonstrated, with tendencies to appear at monolayer and few-layer edges [2].

The nature of such single-photon emitters (SPEs) remains highly debated, ranging from defect centers to local strain or combinations of both [3]. A gap in the understanding encourages correlation of the optical response to the origin of the SPEs. It is thus critical to understand the structural variation at the scale of atomic defects, such as isolated vacancies, through to the nanometer scale with local strain and bending of atomically-thin TMDC layers. Aberration-corrected and monochromated scanning transmission electron microscopy (STEM) is the platform capable of bridging the nanometric excitonic absorption and emission behavior to the nano- and atomic-scale structural properties.

Atomically-thin WSe2 flakes have been mechanically exfoliated from a bulk synthetically-grown crystal, encapsulated by $h$-BN (top and bottom layers of $\sim 15$ and $25 \mathrm{~nm}$ thickness, respectively) in a similar manner as ref. [4,5], and the heterostructure prepared directly on a $\mathrm{Si}_{3} \mathrm{~N}_{4} \mathrm{TEM}$ grid with periodic micronsized holes. Low-temperature $(\mathrm{T} \approx 150 \mathrm{~K})$ cathodoluminescence $(\mathrm{CL})$ spectroscopy and monochromated electron energy-loss spectroscopy (EELS) is measured on a modified Nion HERMES-S200 (ChromaTEM) operated at $60 \mathrm{kV}$. Fig. 1(b) shows the typical CL emission from the encapsulated WSe2 monolayer, including the neutral A exciton $\left(\mathrm{X}^{0}\right)$ and the trion $\left(\mathrm{X}^{-}\right)$peaks well-separated in energy, with the latter $\sim 30 \mathrm{meV}$ lower in energy. $\mathrm{X}^{0}$ and $\mathrm{X}^{-}$show similar intensity variations spatially [Fig. 1(c)], and typically exhibit small shifts of up to $\sim 8 \mathrm{meV}$ within regions of 100 s of $\mathrm{nm}^{2}$. Notably, lower-energy emission lines $\left(\mathrm{X}_{\mathrm{L}}\right)$ demonstrating nanometer-scale localization [Fig. 1(c)] reminiscent of localized SPEs in $h$-BN [6], were also routinely observed. Comparison to PL highlights that such lower-energy lines overlap in energy with the broad defect band and the bandwidth of SPEs typically observed in WSe2. 
The corresponding excitonic absorption of these regions was also measured using monochromated STEMEELS. The four excitonic resonances illustrate a good match to those measured by optical absorption of a similar sample [Fig. 1(d)]. The correlated emission and absorption spectral profiles in Fig. 2(d) reflect that the $\mathrm{X}_{\mathrm{A}}$ absorption energy trends similarly with the $\mathrm{X}^{0} \mathrm{CL}$ emission energy. Furthermore, the Stokes shift, i.e. the energy difference between absorption and emission of the A-exciton transition, was determined to vary in the range of 5-15 meV in different regions [Fig. 1(b), 2(e)]. This is consistent with the reported Stokes shift from PL and optical absorption measurements for WSe2 monolayers [7]. Notably, higher Stokes shift is measured in the redshifted bottom area shown in Fig. 2(d,e). The effect of local strain on the emission and absorption energy, as well as the Stokes shift [7], from nanobeam electron diffraction will also be discussed [8].
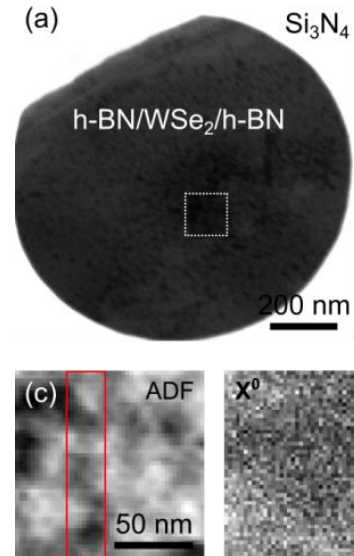
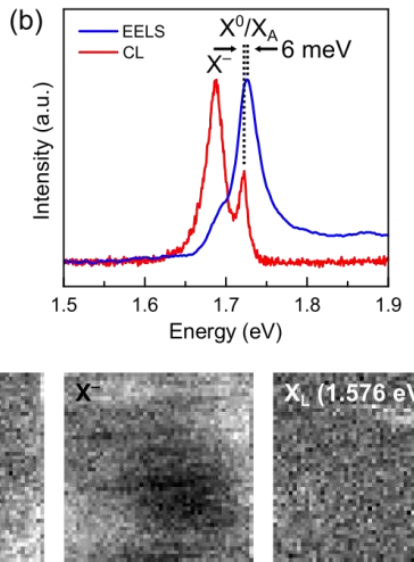
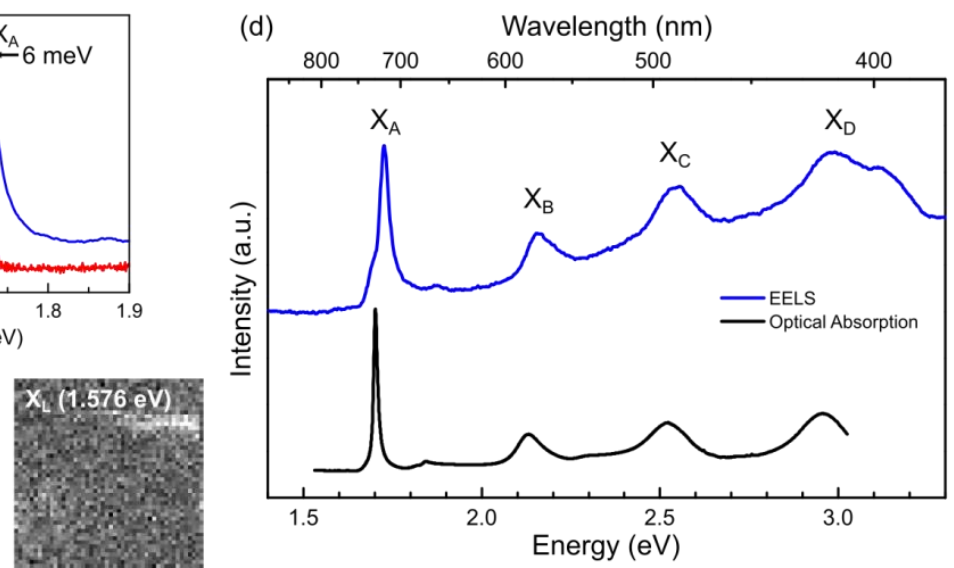

Figure 1. (a) STEM-HAADF image of the h-BN/WSe2 monolayer/h-BN heterostructure with the area of the measurements marked in dotted lines. (b) EELS and CL spectra of the A-exciton from the same region showing a Stokes shift of $6 \mathrm{meV}$. (c) Energy-filtered CL intensity map of the neutral exciton (X0), trion $(\mathrm{X}-)$, and the spatially-localized low-energy (XL) emission. (d) Spectra comparing EELS and optical absorption of h-BN encapsulated WSe 2 at $150 \mathrm{~K}$. 

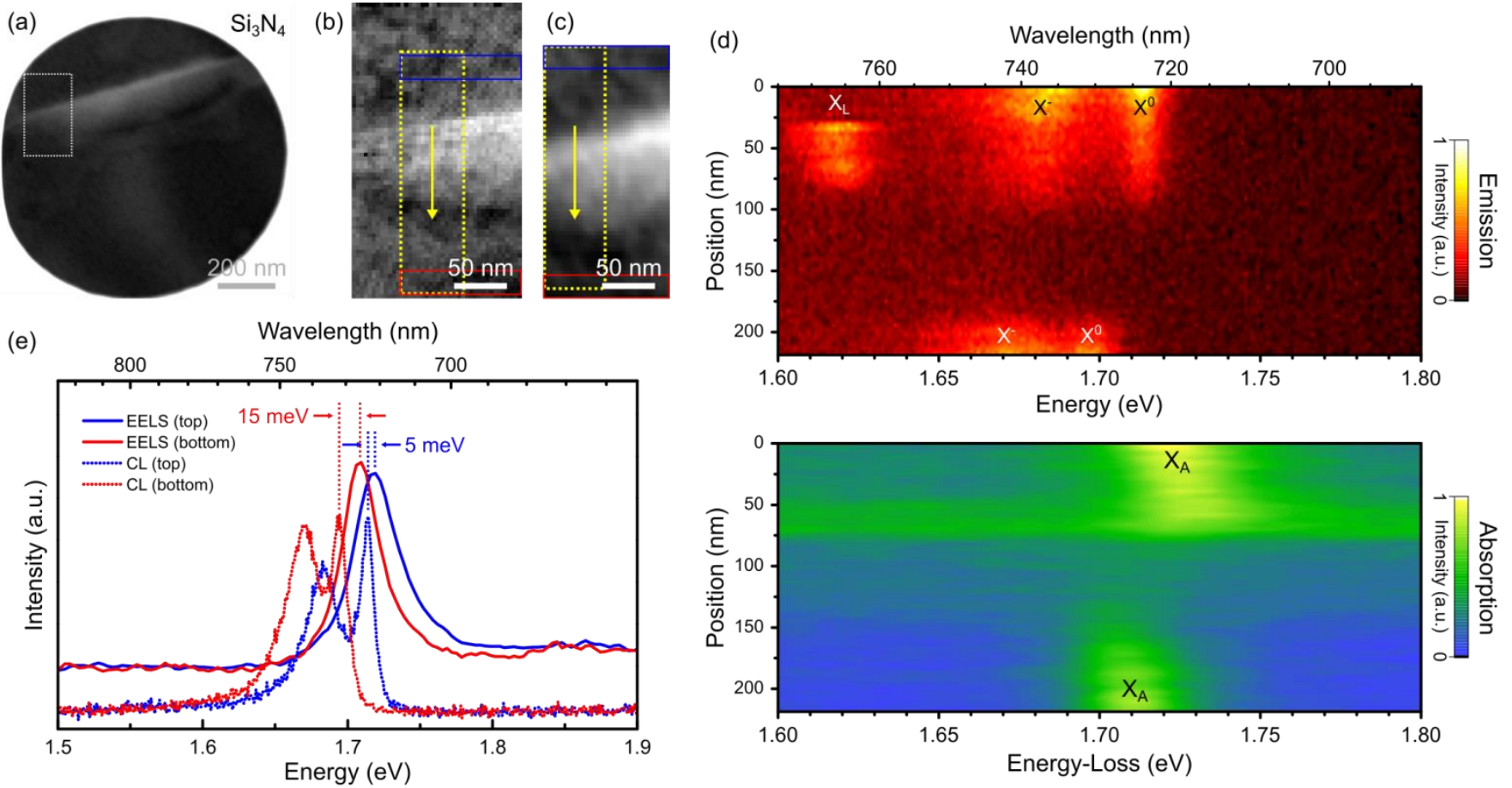

Figure 2. (a) STEM-HAADF image of the heterostructure with the area of the EELS measurement in (b) marked in white dotted lines. Concurrent ADF image acquired for (b) EELS, and (c) CL spectrum images, with the locations of the spectral line profiles in (d) marked in yellow dotted lines, and the EELS and CL spectra in (e) summed in regions marked by color-coded solid lines. (d) EELS absorption (bottom) and CL emission (top) spectral profiles along the yellow arrow direction in (b) and (c), respectively. (e) EELS and CL spectra from regions at the top and bottom of the spectrum images, highlighting the relative redshift for both signals, as well as a higher Stokes shift in the bottom region. The EELS spectra have been offset vertically for clarity.

\section{References}

[1] Jones, A.M., Yu, H., Ghimire, N. et al. Nature Nanotech. 8, 896-869 (2013).

[2] Single photons for all. Nature Nanotech. 10, 481 (2015).

[3] Tonndorf, P., Schmidt, R., Schneider, R. et al. Optica 2, 347 (2015).

[4] Zheng, S., So, J.-K., Liu, F., Liu, Z., Zheludev, N. and Fan, H.J. Nano Lett. 17, 6475-6480 (2017).

[5] Bonnet, N., Lee, H. Y., Shao, F. et al. Nanoscale modification of WS2 trion emission by its local electromagnetic environment. ArXiv: 2102.06140 (2021).

[6] Bourrellier, R., Meuret, S., Tararan, A., Stéphan, O., Kociak, M., Tizei, L.H.G. and Zobelli, A. Nano Lett. 16, 4317-4321 (2016).

[7] Niehues, I., Marauhn, P., Deilmann, T., Wigger, D., Schmidt, R., Arora, A., de Vasconcellos, S.M., Rohlfing, M. and Bratschitsch, R. Nanoscale 12, 20786-20796 (2020).

[8] The authors acknowledge funding from the ANR, program of future investment TEMPOSCHROMATEM (No. ANR-10-EQPX- 50), and the European Union in the Horizon 2020 Framework Program (H2020-EU) under Grant Agreement No. 823717 (ESTEEM3). 